ARTICLE

Received 12 Mar 2014 | Accepted 14 Jul 2014 | Published 22 Aug $2014 \quad$ DOl: 10.1038/ncomms5684 OPEN

\title{
Strong effects of genetic and lifestyle factors on biomarker variation and use of personalized cutoffs
}

\author{
Stefan Enroth, Åsa Johansson ${ }^{1,2}$, Sofia Bosdotter Enroth ${ }^{3} \&$ Ulf Gyllensten ${ }^{1}$
}

Ideal biomarkers used for disease diagnosis should display deviating levels in affected individuals only and be robust to factors unrelated to the disease. Here we show the impact of genetic, clinical and lifestyle factors on circulating levels of 92 protein biomarkers for cancer and inflammation, using a population-based cohort of 1,005 individuals. For $75 \%$ of the biomarkers, the levels are significantly heritable and genome-wide association studies identifies 16 novel loci and replicate 2 previously known loci with strong effects on one or several of the biomarkers with $P$-values down to $4.4 \times 10^{-58}$. Integrative analysis attributes as much as $56.3 \%$ of the observed variance to non-disease factors. We propose that information on the biomarker-specific profile of major genetic, clinical and lifestyle factors should be used to establish personalized clinical cutoffs, and that this would increase the sensitivity of using biomarkers for prediction of clinical end points.

\footnotetext{
${ }^{1}$ Department of Immunology, Genetics, and Pathology, Biomedical Center, SciLifeLab Uppsala, Uppsala University, SE-75108 Uppsala, Sweden. ${ }^{2}$ Uppsala Clinical Research Centre, Uppsala University, SE-75237 Uppsala, Sweden. ${ }^{3}$ Department of Medical Sciences, Uppsala University, SE-75185 Uppsala, Sweden. Correspondence and requests for materials should be addressed to U.G. (email: ulf.gyllensten@igp.uu.se).
} 
A number of protein biomarkers are used for diagnosis and management of cancers and other diseases. Examples include prostate-specific antigen ${ }^{1}$ used to screen for prostate cancer, the ovarian cancer-related tumour marker CA125 (ref. 2) and IL-6, which is a drug target in rheumatoid arthritis (RA) ${ }^{3}$. Ideal biomarkers for early diagnosis should be uniquely present, or overexpressed, in the malignant tumour or blood and not influenced by confounding factors. Most current biomarkers have a function in the normal cell, taking part in, for example, signalling pathways, controlling growth, apoptosis and/ or inflammation ${ }^{4}$. These are not uniquely expressed in the malignant tissue and their expression level is affected by a number of factors, such as the individual's genetic and physical constitution, lifestyle and medication. A detailed understanding of potential confounding factors and their effect size is therefore a necessary prerequisite in the evaluation of the rapidly growing number of candidate biomarkers ${ }^{5}$. The discovery of putative biomarkers for early identification and management of cancer has been greatly facilitated by high-throughput, genome-wide assays. Gene expression analyses have discovered numerous genes that are differentially expressed between malignant and benign tissues $^{6}$, but few have proven suitable as biomarkers, mainly because the mRNA levels do not correlate well with protein abundance ${ }^{7}$. Large-scale studies of protein abundance, on the other hand, have been hampered by lack of high-throughput methods. High-resolution mass spectrometry can be used to examine the underlying genetic contribution to profiles of circulating proteins and effect of environmental covariates, but the resolution is limited by the peptide spectra used and detection sensitivity ${ }^{8}$. An alternative is to use antibody-based measurements, which targets individual, preselected, sets of proteins.

Here we aim to understand the factors that influence normal variation in plasma levels of established and potential biomarkers for cancer, autoimmune diseases and inflammation with the specific goal to facilitate the establishment of individualized clinical cutoffs. To this end, we use the highly sensitive and specific proximity extension assay (PEA) ${ }^{9}$ to estimate the abundance of 92 established or potential biomarkers in plasma from 1,005 individuals from a longitudinal cross-sectional population-based study in Sweden. The biomarkers we analyse here constitute a research panel directed against multiple cancers and also contain proteins implicated in autoimmune diseases such as RA and Graves' disease. PEA combines two dedicated antibodies with a real-time quantitative PCR ( $\mathrm{PPCR}$ ) reaction to achieve high specificity and a wide dynamic range. This technology can be multiplexed without introducing crosstalk, while still maintaining its high specificity and sensitivity ${ }^{10}$. We first determine the effect of a wide range of clinical variables and lifestyle factors, including age, sex, blood pressure, blood group or body mass index (BMI), medication and smoking, on biomarker levels. Then we study the heritability of each biomarker, and by using high-resolution genetic singlenulecotide polymorphism (SNP) array data and whole-exome sequencing, we perform a genome-wide association study (GWAS) for each biomarker. This study is the first to measure biomarker abundance on a large scale using a single technology in a general population, in order to identify contributing factors to normal variation. To our knowledge, only one previous study has studied the genetic association of multiple proteins in the general population. Melzer et al. ${ }^{11}$ investigated 42 proteins using a variety of assays, prohibiting protein-to-protein comparisons, and also did not investigate the protein-specific profiles of covariates. Here by integration of genetic, clinical and lifestyle data, we identify the set of biomarker-specific factors that can be used to determine appropriate individual clinical cutoffs, and thereby enable a more efficient use of each biomarker in personalized cancer management.

\section{Results}

Biomarker measurements. The abundance of 92 proteins (Supplementary Data 1), representing a panel of established and potential biomarkers for cancer and inflammation, were measured in blood plasma of 1,005 individuals from the Northern Sweden Population Health Study (NSPHS), using PEA and qPCR. A total of 77 of the proteins had levels above the detection limit in at least $80 \%$ of our samples, with $91.3 \%(70,651$ of 77,385$)$ of qPCR reactions being successful. In the remaining 15 proteins, $96.8 \%(14,598$ of 15,075$)$ of the protein levels were below the detection limit. Also, $96.5 \%$ (970 of 1,005) of our samples passed quality control on an individual level. The abundance and distribution of the normalized measurements (delta delta $\mathrm{Cq}$ (ddCq)-values) of all the proteins in all samples are illustrated in Fig. 1a, with estimates under the detection limits coloured white. Details on normalization and initial quality control are given below in the Methods section. The proteins with little or no measurable abundance in our samples were: stromelysin-1, GM-CSF (granulocyte-macrophage colony-stimulating factor), estrogen receptor, CA242 (cancer antigen 242), IL-2 (interleukin-2), epiregulin, betacellulin, IL-4, interferon- $\gamma$, IL-7, TNF (tumour necrosis factor), CEA (carcinoembryonic antigen-related cell adhesion molecule 5), MYD88 (myeloid differentiation primary response protein MyD88), mucin-16 and regenerating islet-derived protein 4 . It cannot be ruled out that storage-time and protein degradation could be an influencing factor for these 15 proteins, and previous studies have quantified this specifically for CEA ${ }^{12}$.

Epidemiological associations. To study the effect of clinical and lifestyle factors, we selected 158 phenotypic covariates, including age, sex, blood pressure, BMI, tobacco use, medication, lifestyle (occupation) and sample collection round (2006 or 2009) from the comprehensive set of clinical data available for NSPHS. A multiple linear regression model showed a total of 18 phenotypic covariates to have a significant effect $(P$-value $<0.05$, Bonferroni adjusted) on one or more of 52 of the 77 proteins (Table 1). Factors such as age or weight influenced a broad range of proteins, whereas medication affected specific proteins (Fig. 1b). Notably, smoking affected two proteins, WFDC2 (WAP four-disulfide core domain protein 2) and IL-12, whereas the traditional Swedish moist tobacco product, 'snus' did not have any significant effects, in line with a previous study on effects of tobacco use on DNA methylation ${ }^{13}$. We also found large effects (nominal $P$-value ranging from $1.8 \times 10^{-4}$ to $2.3 \times 10^{-7}$ ) of ABO blood group on three proteins; E-selectin, PECAM-1 (platelet endothelial cell adhesion molecule) and TIE2 (angiopoietin-1 receptor). The connection between E-selectin and blood groups is known ${ }^{14,15}$, but the effect on PECAM-1 and TIE2 has not been described previously. The medication in NHPHS had been investigated using a questionnaire and the reported medications were annotated using the Anatomical Therapeutic Chemical (ATC) classification system. Among the commonly used medications, dihydropyridine derivatives (ATC: C08CA, 54 users), often used to treat hypertension, were correlated to increased IL-6 levels, whereas glucocorticoids (ATC: R03BA, 26 users) lowered both Basigin and hepatocyte growth factor (HGF) receptor levels. Apart from C08CA, no other hypertensive treatment was correlated with high IL-6 levels. Interestingly, the usage of selective $\beta$-2-adrenoreceptor agonist (ATC: R03AC, 13 users), which is commonly found in asthma inhalators, decreased the level of circulating vascular endothelial growth factor D (VEGF-D), which is implicated in the metastasis 

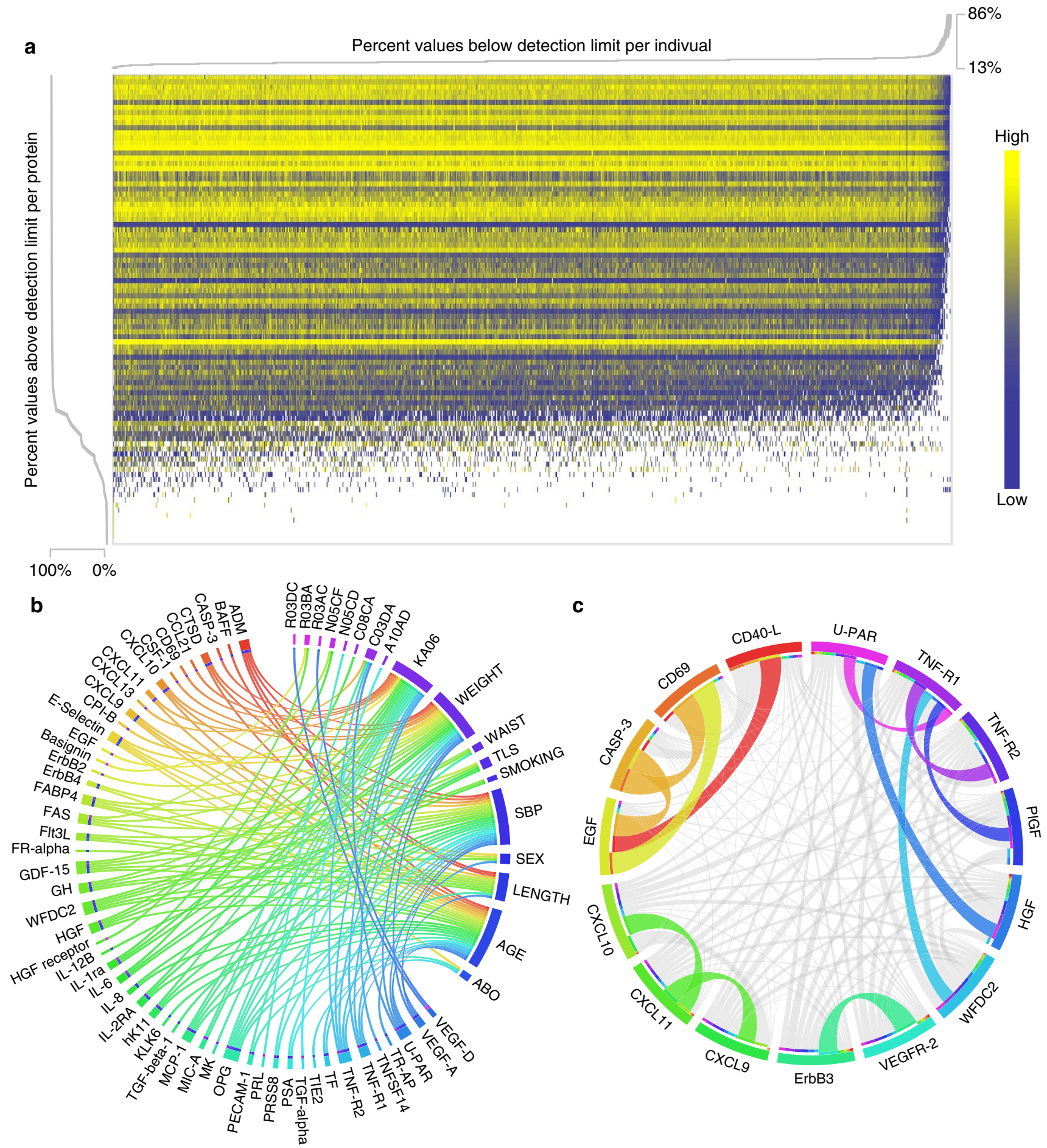

Figure 1 | Characteristics of the PEA measurements. (a) Intensities of PEA values and proportion of proteins and individuals above detection limit. In the heatmap, individuals are in columns and proteins are in rows. Heatmap colours represent ddCq-values ranging from low (blue) to high (yellow) with measurements below detection limit coded white. (b) Significant covariates in relation to each protein. Covariates are listed from the upper right part of the circle (12 o'clock to 4) and connections illustrate significant ( $P$-value $<0.05$, Bonferroni adjusted) contributions to PEA variance. (c) PEA to PEA correlations, coloured connections represent a correlation coefficient $\left(R^{2}\right)$ greater than 0.5 . The width of the connection reflects the magnitude of the squared correlation coefficients. All correlations coefficients $(R)$ were positive.

of non-small lung cancer ${ }^{16,17}$. A detailed description of all investigated covariates and their association with protein levels is given in Supplementary Data 2. The largest fraction of variance explained by a single clinical or environmental covariate was age, which accounted for $27 \%$ of the variation seen for WFDC2. The influence on WFDC2 of age and smoking has previously been reported ${ }^{18}$, but we found that the fraction of variance explained by smoking in our data to be only $1.7 \%$, which is much less than for systolic blood pressure $(14.3 \%)$ or loop-diuretics (ATC: C03CA, plain sulfonamides, $7.2 \%)$. However, these covariates are not necessarily independent as blood pressure and use of medication is related to age. 


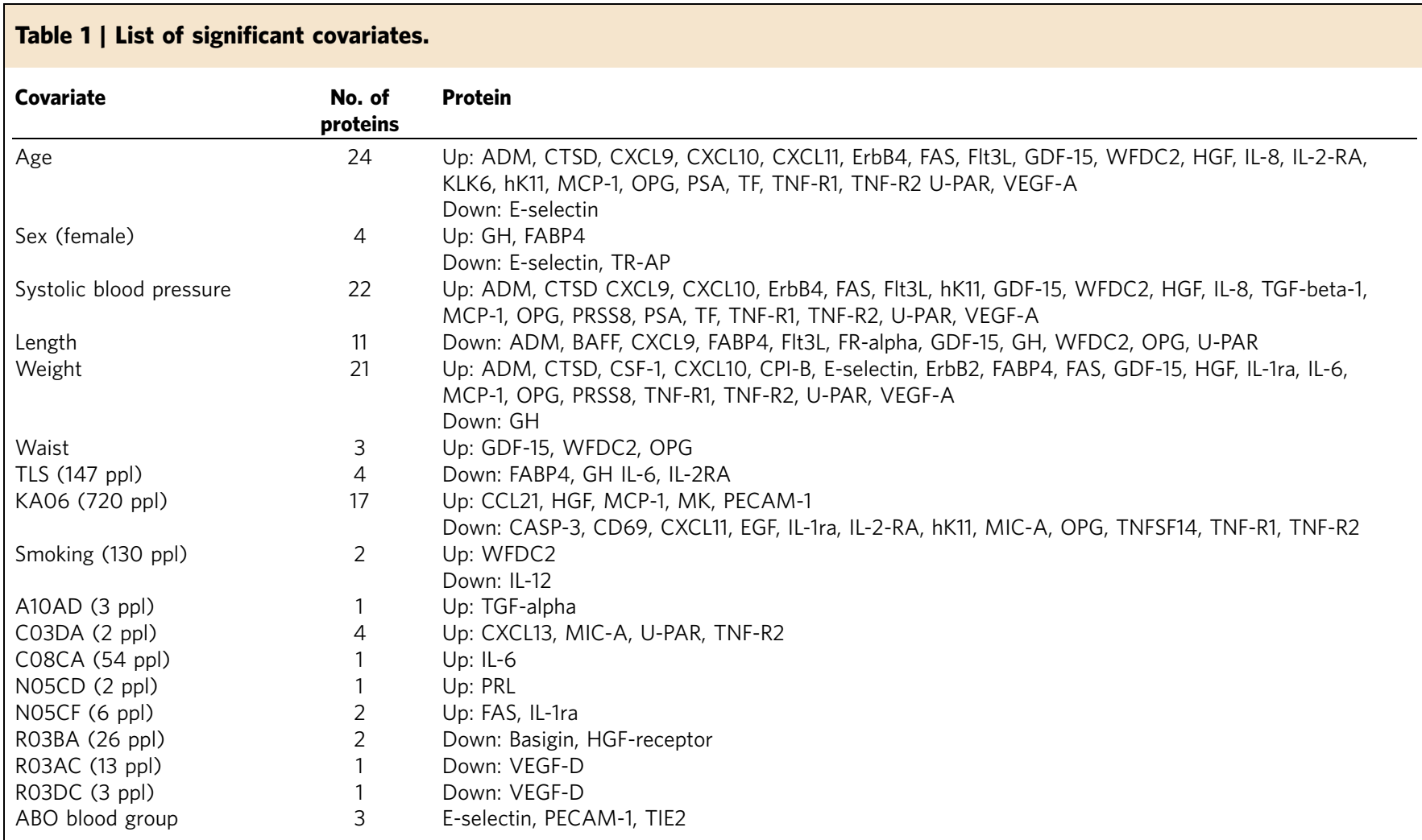

TLS, traditional lifestyle.

Direction of correlations was calculated using PEA-values without any additional covariate correction having been carried out. A10AD (insulins and analogues for injection, intermediate-acting combined with fast-acting), C03DA (aldosterone antagonists), C08CA (dihydropyridine derivatives), N05CD (benzodiazepine derivatives), N05CF (benzodiazepine-related drugs), R03BA (glucocorticoids),

R03AC (selective $\beta$-2-adrenoreceptor agonists), R03DC (leukotriene receptor antagonists).

Correlations between biomarkers. Inter-biomarker correlation was investigated using abundance levels adjusted for significant clinical and lifestyle covariates. These was then rank-transformed into normally distributed values and used to identify 12 pairs with a Spearman's Rho $R^{2}$ greater than 0.5 (Fig. 1c). The highest correlation was found between CASP-3 (caspase-3) and CD69 (early activation antigen CD69; $R^{2}=0.85$ ). CASP-3 was also highly correlated with epidermal growth factor $\left(R^{2}=0.81\right)$, which in turn was highly correlated with $\operatorname{CD} 69\left(R^{2}=0.78\right)$. The strong correlation between some of the biomarkers does not appear to be reflected at the transcription levels. For instance, the Illumina Body Map ${ }^{19-21}$ suggests that CD69 and caspase-3 both are expressed in leukocytes, lymph nodes and adrenal glands (for example, 3 of 16 investigated tissues). In data from leukocytes of 80 controls $^{22}$, there was only a weak correlation between the expression levels of CD69 and CASP-3 $\left(R^{2}=0.13\right)$, suggesting that the high correlation observed at the protein level is either because of post transcriptional regulation, for example, epigenetic regulation, or owing to expression patterns in distinct cell types. Several of the 12 pairs that were highly correlated were proteins with similar functions, such as $\mathrm{C}-\mathrm{X}-\mathrm{C}$ motif chemokine (CXCL)-9, -10, -11, and TNF-R1 and TNF-R2, whereas in other cases apparently unrelated proteins were highly correlated. These correlations may reflect as yet unknown patterns of co-regulation, and bring into question their value as independent biomarkers.

Heritability and genetic association. All 970 individuals' samples that passed the quality control (QC) were used to estimate the heritability for the 77 proteins with measurable levels by evaluating the co-segregation of the protein levels with the relatedness among individuals using a polygenic model (see Methods for details). In $75 \%$ (58 out of 77 ) of the proteins, the levels were found to be heritable (Bonferroni-adjusted $P$-value $<0.05$ ), with heritability ranging from 0.19 to 0.78 and the highest heritability for CCL24 (C-C motif chemokine 24; Supplementary Data 2). Thus, for a majority of the protein biomarkers, circulating levels are significantly affected by the individual's genetic constitution. To determine the nature of the genetic effects on protein abundance, we performed association analyses using over 4.8M SNPs and INDELs identified by direct genotyping and whole-exome sequencing, followed by highquality imputation. In this analysis, each of the 77 proteins was adjusted for the significant clinical and lifestyle variables (Table 1) and the samples were split into a discovery and a replication cohort based on sample collection round (see Methods for details). In the discovery phase, we identified 15 proteins with genome-wide significant hits (nominal $P$-value down to $1.1 \times 10^{-40}$, Table 2), employing a Bonferroni-corrected $P$-value cutoff of 0.05 . Of these, 14 had at least one replicated association (nominal $P$-value down to $1.1 \times 10^{-20}$, Table 2). In all, 175 genome-wide significant hits were detected in the discovery phase, out of which 101 replicated. A combined analysis of all individuals revealed a total of 226 genome-wide significant hits in 14 proteins, with $P$-values down to $4.4 \times 10^{-58}$, and a single marker explaining as much as $26.6 \%$ of the phenotypic variation seen after adjusting for the significant clinical and lifestyle factors (Table 2). A detailed description of each of the 226 hits, including overlaps with previous associations with any phenotype or trait, is given in Supplementary Data 3. IL-6RA (IL-6 receptor subunit alpha) showed the strongest association and the association was caused by one or very few SNPs located in the gene that encodes the respective protein, similar to the case for the majority of the biomarkers (Fig. 2a). Conditioning on the top-hit revealed that four of the proteins, CCL24, MIC-A 
Table 2 | GWAS results.

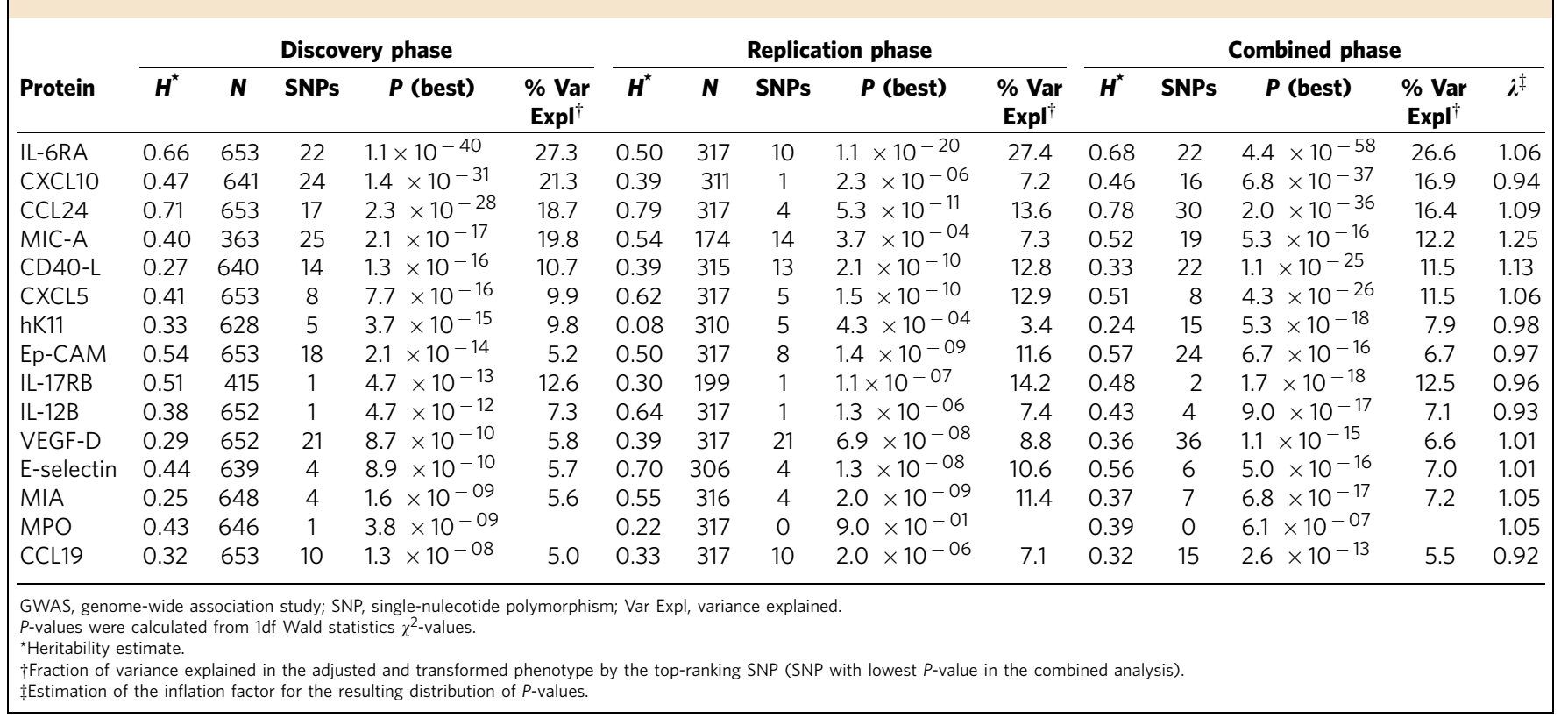
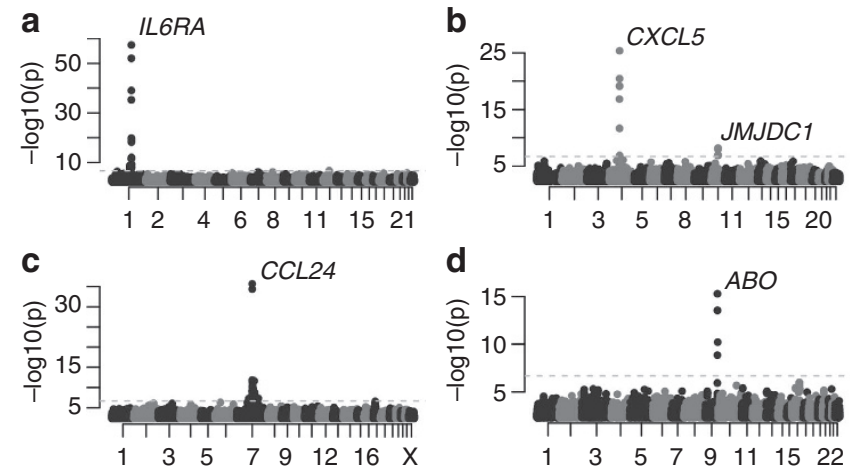

Figure 2 | Manhattan plots of GWAS results. (a) IL-6RA (b) CXCL5

(c) CCL24 and (d) E-selectin. $X$ axis labels refer to human chromosomes listed 1-22 and X. P-values were calculated from 1df Wald statistics $\chi^{2}$ values using 971 individuals.

(major histocompatibility complex class I polypeptide-related sequence A), CXCL5 and Ep-CAM (epithelial cell adhesion molecule), had hits independent of the highest-ranking SNP (Table 3). For CXCL5 (Fig. 2b) and Ep-CAM, the second SNP was located on a different chromosome, whereas for CCL24 (Fig. 2c) and MIC-A, the second SNPs were located close $(<40 \mathrm{~kb}$, Table 3$)$ to the first hit. The second SNP for Ep-CAM explained $6.5 \%$ of the variance of the unadjusted phenotype, as compared with $4.9 \%$ for the top-ranking SNP. For the other three proteins, the fraction of variance explained by the second-ranking SNPs was small compared with the top-ranking SNP. For 12 of the 14 biomarkers with a strong genetic association (CCL24, CD40-L (CD40 ligand), CXCL5, CXCL10, Ep-CAM, IL-12B, IL-17RB (IL-17 receptor B), IL-6RA, hK11 (Kallikrein-11), MIA (melanoma-derived growth regulatory protein), MIC-A and VGEF-D), the top SNPs were located in cis with the gene encoding the protein. We compared our 226 hits with eQTLs as reported by the NCBI's eQTL database and found overlapping SNPs in 11 cases. These were reported for IL-17RB (one SNP) and CCL24 (one SNP) in liver ${ }^{23}$ and for MIC-A (nine SNPs) in lymphoblastoids ${ }^{24}$ (Supplementary Data 3). As expression is cell type specific and eQTL studies only exist for a limited set of tissues, the number of SNPs found here to be eQTL is likely to be an underestimate. For two of the proteins (CCL19 and E-selectin), the genome-wide significant hits were located at other loci than the one coding for the protein (Table 3). The top hits for CCL19 were located in the major histocompatibility complex class II gene cluster, encoding molecules present on antigen-presenting cells and B-cell lymphocytes. CCL19 is a chemokine implicated in inflammatory and immunological responses, but also in normal lymphocyte recirculation and homing. Higher serum levels of CCL19 have been associated with poor prognostics of AIDS patients $s^{25}$. For E-selectin, the circulating level is known to be affected by $\mathrm{ABO}$ blood group. Here, even after correction for blood group at the $\mathrm{A} / \mathrm{B} / 0$-level, the top hits in the GWAS were located within the $A B O$-gene, determining the blood group (Fig. 2d), with our top hit (rs507666) being a perfect tag SNP for the A1 subtype ${ }^{26}$, suggesting that the specification of the A group into A1 and A2 is involved. Our dependency of the E-selectin levels on $\mathrm{ABO}$ status is consistent with the pattern described earlier ${ }^{15}$, where individuals with the $\mathrm{O}$ blood type have the highest levels. This is in contrast to the patterns for TIE2 and PECAM-1, where individuals carrying the $\mathrm{B}$ or $\mathrm{AB}$ blood group have the highest values (Supplementary Fig. 1). For the other proteins (Ep-CAM, CCL19 and CXCL5), we found no evidence such as eQTLs or common pathways linking the loci that did not code the protein to the gene coding the protein. In summary, for a large number of the biomarkers, significant genetic effects on protein levels could be identified.

Personalized biomarker-specific covariate profiles. The relative importance of individual genetic, clinical and lifestyle factors on the abundance differed dramatically between the 77 biomarkers (Fig. 3a). Some biomarkers were affected by strong genetic factors, whereas others mainly by environmental or clinical factors. These variables are not always independent, such as blood pressure and use of medication, which are both related to age. This can be seen in that the total fraction of observed variance, as determined by a combined model including all 158 covariates plus the top-ranking SNP and the top-ranking SNP from the 
Table 3 | Location and annotation of top GWAS hits.

\begin{tabular}{|c|c|c|c|c|c|c|c|}
\hline Protein & SNP & P-value & Effect, $\beta$ (s.e.) & Effect allele (reference) & chr:position ${ }^{\star}$ & Gene & Type \\
\hline IL-6RA & rs4129267 & $4.39 \times 10^{-58}$ & $0.84(0.052)$ & $\mathrm{T}(\mathrm{C})$ & $1: 154426264$ & IL6RA & Intronic \\
\hline CXCL10 & rs11548618 & $6.78 \times 10^{-37}$ & $1.80(0.14)$ & $A(G)$ & $4: 76943947$ & CXCL10 & Nonsynonymous \\
\hline \multirow[t]{2}{*}{ CCL24 } & rs6946822 & $2.02 \times 10^{-36}$ & $-0.62(0.049)$ & $\mathrm{T}(\mathrm{C})$ & $7: 75479448$ & CCL24 & Intergenic, 36 kb upstream \\
\hline & rs11465293 & $7.95 \times 10^{-13}$ & $-0.63(0.088)$ & $A(G)$ & $7: 75442723$ & CCL24 & Nonsynonymous \\
\hline \multirow[t]{2}{*}{ MIC-A } & rs3869132 & $5.33 \times 10^{-16}$ & $-0.73(0.090)$ & $A(G)$ & $6: 31410948$ & MIC-A & Intergenic, 28 kb downstream \\
\hline & rs2263316 & $1.02 \times 10^{-08}$ & $0.48(0.083)$ & $G(A)$ & $6: 31421297$ & MIC-A & Intergenic, 38 kb downstream \\
\hline CD40-L & rs148594123 & $1.07 \times 10^{-25}$ & $-0.96(0.091)$ & $A(G)$ & $X: 135741443$ & CD40LG & Nonsynonymous \\
\hline \multirow[t]{3}{*}{ CXCL5 } & rs425535§ & $4.27 \times 10^{-26}$ & $-0.86(0.081)$ & $\mathrm{T}(\mathrm{T})$ & $4: 74863997$ & CXCL5 & Synonymous \\
\hline & rs2472649ll & $3.57 \times 10^{-21}$ & $-0.70(0.074)$ & $A(A)$ & $4: 74857708$ & CXCL5 & Intergenic, $4 \mathrm{~kb}$ downstream \\
\hline & rs2393967† & $4.54 \times 10^{-08}$ & $0.30(0.052)$ & $C(A)$ & $10: 65133156$ & JMJD1C & Intronic \\
\hline hK11 & rs117268623 & $5.25 \times 10^{-18}$ & $-1.48(0.17)$ & $\mathrm{T}(\mathrm{C})$ & $19: 51527970$ & $K L K 11$ & Nonsynonymous \\
\hline \multirow[t]{2}{*}{ Ep-CAM } & rs201314303§ & $6.74 \times 10^{-16}$ & $-2.48(0.31)$ & $G(C)$ & $2: 47612302$ & EPCAM & Intronic \\
\hline & rs56398830 & $1.26 \times 10^{-15}$ & $-0.94(0.12)$ & $A(G)$ & 13:103701690 & SLC10A2 & Nonsynonymous \\
\hline \multirow[t]{2}{*}{ IL-17RB } & rs6801605 & $1.75 \times 10^{-18}$ & $-0.56(0.064)$ & $A(G)$ & $3: 53876218$ & $\mathrm{CHDH}$ & Intronic \\
\hline & & & & & & IL17RB & 4 kb upstream \\
\hline IL-12 & rs10045431 & $8.99 \times 10^{-17}$ & $0.47(0.057)$ & $A(A)$ & $5: 158814533$ & IL12B & Intergenic, 57 kb upstream \\
\hline \multirow[t]{2}{*}{ VEGF-D } & rs188779336§ & $1.11 \times 10^{-15}$ & $-1.58(0.20)$ & $G(C)$ & X:15308292 & ASB11 & Intronic \\
\hline & rs146086561 & $1.81 \times 10^{-15}$ & $-1.57(0.20)$ & $\mathrm{T}(\mathrm{C})$ & $X: 15365438$ & FIGF & Nonsynonymous \\
\hline E-selectin & rs507666 & $5.01 \times 10^{-16}$ & $-0.55(0.067)$ & $A(G)$ & 9:136149399 & $A B O$ & Intronic \\
\hline \multirow[t]{4}{*}{ MIA } & rs2230694\$ & $6.83 \times 10^{-17}$ & $0.66(0.079)$ & $G(A)$ & $19: 41263403$ & SNRPA & Synonymous \\
\hline & rs2607426" & $1.13 \times 10^{-16}$ & $0.65(0.079)$ & $G(A)$ & $19: 41274713$ & MIA & Intergenic, 6k upstream \\
\hline & rs2233154 ",\$ & $1.13 \times 10^{-16}$ & $0.65(0.079)$ & $\mathrm{T}(\mathrm{C})$ & $19: 41281346$ & MIA & UTR5 \\
\hline & rs2233159 ${ }^{\prime \prime} \$$ & $1.07 \times 10^{-16}$ & $-0.65(0.079)$ & $C(C)$ & $19: 41283365$ & MIA & UTR3 \\
\hline CCL19 & rs7775228 & $2.63 \times 10^{-13}$ & $0.51(0.069)$ & $C(T)$ & $6: 32658079$ & $H L A-D Q B 1$ & Intergenic, 24 kb upstream \\
\hline $\begin{array}{l}6.7 \times 10^{-5} \text { fo } \\
\text { \$lmputed. }\end{array}$ & $\begin{array}{l}\text { not discovered ir } \\
\text { e discovery and }\end{array}$ & $\begin{array}{l}\text { statistics } \chi^{2} \text {-valt } \\
\text { ci as per condit } \\
\text { nconditional dis } \\
\text { tion cohorts, res }\end{array}$ & $\begin{array}{l}\text { ry-replication analys } \\
\text { ively. }\end{array}$ & was subse & onal discover & tricury & sulting in the $P$-values $3.9 \times 10^{-9}$ and \\
\hline
\end{tabular}

conditional analysis, lie between 0.20 and 0.56 (Fig. 3b), whereas the sum of the explained variance by individual covariates in some cases reached above 1 (Fig. 3a). Figure 3 illustrates the main results of the study. Most of the 77 biomarkers showed large variation in abundance between individuals but they differed considerably with regard to the specific genetic, clinical or lifestyle factors involved. At one extreme, IL-6RA levels were affected most strongly by the individuals' genotype and only a very small fraction of the variance was explained by other covariates, with BMI being the strongest (1.0\%). Even assuming that all 158 covariates, besides the top ranking SNP, contributed independent effects, the sum of the fraction of the variance of IL-6RA levels explained by these factors was less $(21.0 \%)$ than the single genetic effect $(21.3 \%)$. At the other end of the spectrum, HGF did not show a significant heritability, and none of the genetic markers reached genome-wide significance. However, 17 other covariates were nominally significant $(P$-value $<0.05)$ for HGF and 4 (weight, sample round, systolic blood pressure and age) remained significant after correction for multiple testing. These covariates accounted for $3.3 \%, 2.9 \%, 14.1 \%$ and $19.3 \%$, respectively, of the percentage-of-variance-explained. In addition, the use of platelet aggregation inhibitors (ATC: B01AC) and loop-diuretics (ATC: C03CA) explained $5.7 \%$ and $7.1 \%$ of the variance observed in the unadjusted ddCq-values, respectively, whereas the top ranking SNP only accounted for $1.6 \%$. In the middle part of the distribution in Fig. 3a, we find biomarkers that were less affected by the genetic, clinical or environmental factors studied, possibly reflecting limited non-disease-related variability.

Information on the set of important variables for each biomarker can be used to reduce the non-disease-related variation. For instance, soluble CXCL10, which shows elevated levels in patients with a number of autoimmune-related diseases ${ }^{27}$, has previously been shown to be associated with systolic blood pressure ${ }^{28}$. Here, we confirm the correlation with systolic blood pressure, which explains $5.6 \%$ or the variability, but we also found a significant correlation with age $(9.0 \%$ of variability) and a very strong effect of genetic variants $(35.4 \%$ of variability). Stratifying individuals on age did not appreciable reduce the range of variability (Fig. 3c). However, stratifying on the basis of the genotype at the top hit ( $r 11548618)$ had a considerable effect on reducing the variability (Fig. $3 \mathrm{~d}$ ). In the case of CCL24, the carriers of the reference allele of rs6946822 had a level 209\% (linearized ddCq) of the average value of the homozygote carriers of the alternative allele (Fig. 3e). The effect of medication on the abundance can be demonstrated by IL-6 (Fig. 3f), where the distribution of protein level was clearly shifted upwards with the use of dihydropyridine derivatives (ATC: C08CA) found in drugs prescribed for treatment of hypertension or angina pectoris. Interestingly, this was the only hypertension medication that is correlated with higher IL-6 levels, and neither angiotensin-converting-enzyme (ACE)-inhibitors (ATC: C09AA), selective $\beta$-blocker agents (ATC: C07AB) nor a combination of these mediate this effect (Fig. 3f). This implies that detailed medication information may be needed for proper use of this biomarker.

Availability. Full summary statistics of the combined results from the 14 GWAS's with genome-wide significant hits are available from doi:10.5879/BILS/g000001.

\section{Discussion}

We have shown that for 72 of the 77 biomarkers studied, the circulating plasma levels are strongly associated with genetic, 


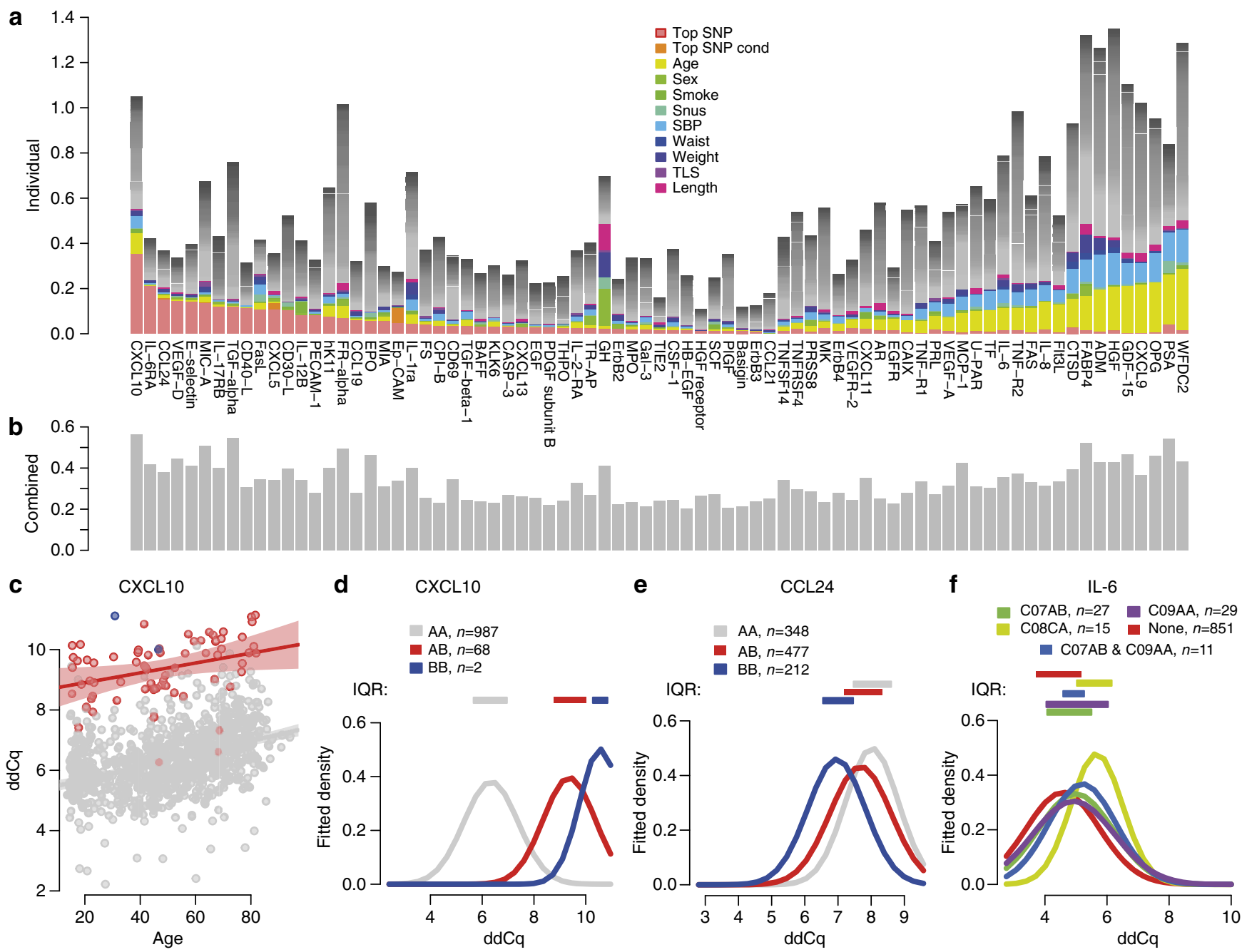

Figure 3 | Covariates and protein biomarkers. (a) Variance explained by each of the covariates for the set of 77 biomarkers with measurable variability with the 11 most important covariates coloured. The combined effect of the remaining covariates is shown in grey, assuming independence in effect between covariates. (b) The percent of the variance explained by the full set of covariates studied for the 77 proteins, using a combined model. (c) Abundance of CXCL10, expressed as ddCq-values, in relation to age when stratified by genotype at rs11548618; AA (grey), AB (red) and BB (blue). Shadowed areas represent the $95 \%$ confidence interval in a linear model predicting ddCa from age. (d) Fitted normal distribution densities based on mean and standard deviation in ddCq-values for CXCL10, split by the rs11548618 genotype. (e) Fitted normal distribution densities based on mean and standard deviation in ddCq-values for CCL24 split by the rs6946822 genotype. (f) Fitted normal distribution densities based on mean and standard deviation in ddCq-values for IL-6 split by use of hypertension medications. Only groups where there are at least 10 individuals are shown. C07AB: $\beta$-blocking agents, selective. C08CA: dihydropyridine derivatives. C09AA: ACE inhibitors, plain. (d-f) Interquartile ranges indicated with coloured boxes above the curves.

clinical or lifestyle factors. Most biomarkers are highly heritable, and for 14 biomarkers, we identified strong genetic associations, with the top SNP explaining as much as $36 \%$ of the variability in protein abundance between individuals. For these biomarkers, stratifying patients based on their genotype may dramatically enhance the ability to detect deviations from normal circulating levels. A number of non-genetic factors also show a strong effect on biomarker levels, with age, systolic blood pressure and weight affecting a large number of the biomarkers. As cancer incidences increase with age ${ }^{29}$, so does the use of prescribed medications (Spearman's rho, $R^{2}=0.29$, Supplementary Fig. 2). Interestingly, we identified medication as an important clinical variable that should be considered when using the biomarkers for diagnosis or risk prediction. For instance, Basigin expression has been associated with shorter survival and proposed as a biomarker for adjuvant therapy in colorectal cancer ${ }^{30}$. Our analysis did not show any significant association of Basigin levels with covariates such as anthropometrics, age, sex or smoking. However, the use of glucocorticoids commonly found in inhalators used to treat asthma-related conditions, decreased circulating levels of Basigin thereby possibly masking the need for adjuvant treatment. Our results indicate that when using Basigin as a biomarker in an ageing population, medication history and dosage should be taken into account in order to establish an appropriate clinical cutoff. Another example is the IL-6 and IL-6 receptor (IL6-RA), where we confirm the strong effect of the genetic constitution on the circulating IL6-RA levels. We also show that medications used to treat, for example, hypertension such as dihydropyridine derivatives, but not ACE-inhibitors or selective $\beta$-blockers agents, cause or maintain an increase in the inflammatory response cascade via high IL-6 levels. The IL-6 signalling is important in the pathogenesis of several autoimmune and chronic inflammatory diseases ${ }^{31}$ and antibody-based drugs are used to target the IL-6 receptor in patients with RA in order to dampen 
the inflammatory response ${ }^{32}$. In clinical practice, only two-thirds of the patients treated with these drugs respond to the treatment and factors such as age and medical history have been shown to be predictors of remission and response in RA patients ${ }^{33}$. Future investigations are clearly needed to specifically address the longterm effects of commonly used medications in this perspective.

In a clinical context, circulating levels of CXCL10 have been estimated to $120 \pm 83 \mathrm{pg} \mathrm{ml}^{-1}$ in patients diagnosed with Graves' Disease as compared with $72 \pm 32 \mathrm{pg} \mathrm{ml}^{-1}$ in controls ${ }^{34}$; an average increase of $67 \%$ not taking genetic and non-genetic covariates into account. By comparison, the average increase in individuals in our study carrying the reference genotype for $\mathrm{rs} 11548618$ was $178 \%$ (linearized ddCq) of the level in heterozygous individuals, clearly illustrating the relative importance of carrier genotype versus to disease state on biomarker levels. Previous efforts ${ }^{35,36}$ have identified genetic susceptibility loci for Graves' disease but none of these overlap with the loci associated with the CXCL10 levels, suggesting that in this case the causal effects of the disease are not directly linked to the biomarker levels. Another strong genetic effect was observed for CCL24, where carriers of the reference allele of rs6946822 have a level 209\% (linearized ddCq) of the average value of the homozygous carriers of the alternative allele (Fig. 3e). The worldwide minor allele frequency of rs6946822 is listed in NCBI's short genetic variation database $(\mathrm{dbSNP})$ as 0.46 , implying that every fifth individual will be homozygote, similar in frequency to the individuals who smoke in the United States today ${ }^{37}$, demonstrating the large, common genetic effects on biomarker variation found in the population today.

We also find biomarkers that are not significantly affected by any of the variables examined, rendering them less susceptible to variability induced by non-disease-related factors. Although we have investigated a large number of genetic, clinical and lifestyle factors, they altogether explain at most $56 \%$ of the variation in biomarker levels between individuals. The remaining variance must reflect other factors, or non-additive interaction between some of the factors studied, and their identification could further increase the utility of biomarkers by reducing sources of variation unrelated to disease state. For example, CCL24 had a heritability of 0.78 , indicating that additional genetic loci might affect protein levels. For 15 of the biomarkers, the vast majority of abundances were below the detection limits in our cohort. Several of these could represent ideal biomarkers without major presence in normal plasma and thus with no influencing genetic or lifestyle factors. Among these was for instance mucin-16 (or CA125) that is used clinically as a test for ovarian cancer ${ }^{38}$ and also potential biomarkers such as regenerating islet-derived protein-4 that has been proposed as a biomarker for pancreatic ductal adenocarcinoma ${ }^{39}$.

This study identifies several previously unknown genetic and lifestyle factors influencing the circulating plasma levels of disease biomarkers in a population-based cohort, but has its limitations. First, we have a relatively small sample size $(N=1005)$ for genetic association studies. Despite this fact, we identify and replicate 12 novel associations of large effect on the disease biomarkers. Although large GWAS consortia have identified hundreds of genetic variants associated with variation in disease-related phenotypes $^{40,41}$, most of these SNPs are common and have such small effect sizes that they are not clinically useful.

Personalized cancer medicine is on a trajectory from long awaited promise to existing reality, with clinical applications for a small number of cancers with directed treatments. In chronic myelogenous leukemia, patients with a specific translocation respond well to treatment with a tyrosine-kinase inhibitor blocking an enzyme that in turns triggers signalling cascades ${ }^{42}$. Also, patients with non-small-cell lung cancer and a gene-fusion mutation have higher drug response rates than those lacking this gene fusion ${ }^{43}$. However, the number of cancer biomarkers in clinical use is still limited. In the set of biomarkers studied here, we identified a surprisingly strong genetic effect on some biomarkers after correcting for clinical (medication) and lifestyle variables. Likewise, other biomarkers were strongly affected by environmental lifestyle or clinical factors. Genotyping of selected polymorphisms with a strong effect on abundance appears to be crucial for about $20 \%$ of the biomarkers in our study, whereas lifestyle and medication are important covariates for the majority. In the daily clinical routine, we envision that analysis of broad-spectrum biomarkers could be used as a follow-up analysis for patients, or for screening of risk groups. Our analysis indicate that such tests would be accompanied by collecting additional relevant information such as anthropometrics, medication and genotyping of specific polymorphisms known to affect the baseline of these biomarkers. The clinical laboratory that performs the biomarker analysis would have documentation on which cofactors that significantly influence the baseline levels, and could advise the physician on how to interpret the outcome of the test. Our results imply that using biomarker-specific covariate profiles will make it possible to determine more precise, individualized, clinical cutoff levels. This in term could lead to a more efficient use of protein biomarkers for early detection of abnormal levels and for increased sensitivity and specificity in disease diagnosis. By employing biomarker-specific profiles of covariates it will be possible to fully harness the potential of existing and novel biomarkers for disease diagnosis and management.

\section{Methods}

Samples. The NSPHS was initiated in 2006 to provide a health survey of the population in the parish of Karesuando, county of Norrbotten, Sweden, and to study the medical consequences of lifestyle and genetics. This parish has about 1,500 inhabitants who meet the eligibility criteria in terms of age ( $\geq 15$ years), of which 719 individuals participated in the study (KA06 cohort). As a second phase of the NSPHS, another 350 individuals from a neighbouring village (Soppero) were recruited in 2009 (KA09 cohort). For each participant in the NSPHS, blood samples were taken (serum and plasma) and stored at $-70{ }^{\circ} \mathrm{C}$ on site. Both the 2006 and 2009 samples used in this study have undergone two freeze-thaw cycles before the measurements carried out here. DNA has been extracted for genetic analyses and detailed descriptions of this study have been published elsewhere ${ }^{44-46}$. A questionnaire was used to collect data on medications and lifestyle. The questionnaire was filled in at the local health-care centre in the presence of the local district nurse. Notably, around $15 \%$ of the participants of the study adhere to a traditional lifestyle based on reindeer heading and crafts. Differences in, for example, diet in this group compared with the group with a lifestyle typical of more industrialized regions have been shown to increase levels of circulating blood lipids ${ }^{47}$, which motivates to include the traditional lifestyle adherence as a covariate.

Ethical considerations. The NSPHS study was approved by the local ethics committee at the University of Uppsala (Regionala Etikprövningsnämnden, Uppsala, 2005:325) in compliance with the Declaration of Helsinki ${ }^{48}$. All participants gave their written informed consent to the study including the examination of environmental and genetic causes of disease. In cases where the participant was not of age, a legal guardian signed additionally. The procedure that was used to obtain informed consent and the respective informed consent form has recently been discussed in light of present ethical guidelines ${ }^{49}$.

Multiplexed PEA. Protein levels in plasma were analysed using the Olink Proseek Multiplex Oncology I $96 \times 96$ kit and quantified by real-time PCR using the Fluidigm BioMark HD real-time PCR platform as described earlier ${ }^{10}$. In brief, for each measured protein, a pair of oligonucleotide-labelled antibodies probes bind to the targeted protein, and if the two probes are in close proximity, a PCR target sequence is formed by a proximity-dependent DNA polymerization event and the resulting sequence is subsequently detected and quantified using standard real-time PCR. Each plate contains 96 wells whereof 92 are samples, 1 is a negative control and 3 are positive controls (spiked in IL-6, IL-8 and VEGF-A). Each sample is also spiked in with two incubation controls (green fluorescent protein and phycoerythrin), one extension control and one detection control. These controls are used to determine the lower detection limit (negative control) and to normalize 
the measurements into ddCq values according to the following formulae

$$
d d C q=d C q_{\text {blank }}-(-)\left(d C q_{\text {analyte }}\right)
$$

where

$$
(-)\left(d C q_{\text {analyte }}\right)=C q_{\text {analyte }}-C q_{\text {Extension Control }}
$$

and $d C q_{\text {blank }}$ is a per-assay value defined by the manufacturer to give a positive $\log 2$-scale. The ddCq values were then $\log 2$-transformed for subsequent analysis. Each PEA measurement has a specified lower detection limit calculated based on negative controls that are included in each run and measurements below this limit were removed from further analysis. Individual samples where at least one of the internal controls contained an outlier value $(n=35)$ or where too many $(>75 \%)$ measurements were below detection limits in any PEA $(n=1)$ were also excluded from further analyses (total $n=35$ of $1005,3.5 \%$ ). We wanted at least 200 observations per protein above detection limit in order to conduct the downstream statistical analyses and therefore proteins with fewer observations were excluded from further analyses. After individual and protein quality control, 77 proteins measured in 970 individuals remained. Out of the removed proteins, seven proteins (betacellulin, epiregulin, IL-2, CA242, estrogen receptor, G-CSF and stromelysin-1) had $100 \%$ of measurements below detection limit. Uniprot recommended short names have been used throughout when these are available otherwise; the assay manufacturers' abbreviations have been used. All assay characteristics including detection limits and measurements of assay performance and validations are available from the manufacturer's webpage (http://www.olink.com/products/ proseek-multiplex/downloads/data-packages).

Genotype data. The KA06 and KA09 cohorts have previously been genotyped on the Illumina Infinium HapMap300v2 BeadChip (308,531 markers; Illumina) and Illumina Human OmniExpress BeadChip (731,442 markers; Illumina) arrays, respectively, as described earlier ${ }^{8}$. In brief, the specific KA06 and KA09 data were quality checked separately leaving 691 individuals with 306,086 SNPs at $99.50 \%$ genotyping rate and 346 individuals 631,503 SNPs at $99.88 \%$ genotyping rate, respectively. Four individuals were present in both cohorts and these were removed from the KA06 data. Here, we also genotyped the individuals from both cohorts ( $n=1059$ ) on the Illumina Human Exome Beadchip containing 247,901 SNPs, insertions and deletions primarily selected to have coding changes. The genotype calling was done with the software GenomeStudio 2011.1 (Illumina Inc.) using a Project Sample generated Cluster File as recommended by the manufacturer. The Exomechip data were quality controlled requiring $95 \%$ and $98 \%$ genotyping rate on marker and individual levels, respectively, and a Bonferroni-corrected HardyWeinberg cutoff of 0.05 leaving 242,519 markers at a total genotyping rate of $99.94 \%$ in the 1,033 unique individuals previously genotyped. This analysis was carried out using custom R-scripts and PLINK (v1.07) ${ }^{50}$.

Exome sequencing. We selected 100 individuals, 68 from KA06 and 32 from KA09, for Whole-Exome Sequencing using Agilent's SureSelect system (Agilent) for exome capture and the SOLiD 5500xl instrumentation for sequencing. Each sample was sequenced to at least $30 \mathrm{X}$ coverage. The individuals were selected to represent as much genetic variation of the cohort as possible ${ }^{51}$. Alignment was done using the LifeScope software, and SNPs and INDELs were called using diBayes. For each position $(n>1.5 \mathrm{M})$ where any individual so far sequenced at the Uppsala Genome Centre had called SNP or INDEL, we then checked our 100 individuals for coverage in order to differentiate between missing and reference calls. These positions were included to maximize the overlap with the 1,000 genomes reference panels to ensure proper imputation using two reference panels. Reference calls for SNPs were made if there were at least three reference sequence reads with unique start points and a maximum of $5 \%$ reads with non-reference at that position. Reference calls for INDELs were made if there was no reads at all without the reference call. All other calls were set to missing. We then required at most 5\% missing call rate per SNP or INDEL. This resulted in 83,568 SNPs with non-zero MAF at $98.74 \%$ total genotyping rate and 38,290 INDELs with a total genotyping rate at $99.45 \%$ and an additional $350 \mathrm{k}$ positions with reference calls only. We then required a genotyping rate of $95 \%$ in both individual and marker level and a Bonferroni-corrected Hardy-Weinberg cutoff at 0.05 , which resulted in 468,630 markers at total genotyping rate of $98.79 \%$.

Imputation of genotype data. We created an in-house reference panel to be used simultaneously with the 1,000 genomes $^{52}$ reference panel ${ }^{53}$. The in-house panel was based on the 100 exomed individuals by merging the SNPs and INDELs called from the exomes with the SNPs common between the Illumina Human HapMap300v2 (used in the KA06 cohort) and the Illumina Human OmniExpress (used in KA09 cohort), $n=182,916$, and all the markers from the Illumina Human Exome chip. In this step, there was no additional filtering done on minor allele frequency in order to maximize the overlap with the SNPs in the 1,000 genome panel. The total number of markers in the in-house reference panel was 847,855 . The reference haplotypes were created using in-house R-scripts, PLINK (v1.07) and phased using SHAPEIT (v2.r) ${ }^{54}$. Data were then imputed for the two cohorts separately using IMPUTE2 (v2.3.0) with a pre-phasing approach ${ }^{55}$. The input data were phased chromosome-wise using SHAPEIT (v2.r). In addition to our in-house panel, we also utilized the 1,000 Genomes Phase I integrated variant set (National Center for Biotechnology Information build b37, March 2012) accessed from the IMPUTE Web resource ${ }^{53}$. IMPUTE2 was run with the default parameters with the following changes '-- merge-ref-panels' and '-k_hap 500 200'. The latter instructing IMPUTE2 to use 500 haplotypes from the $1,000 \mathrm{G}$ reference panel and all 200 from our in-house panel. Data were imputed in chunks of around $5 \mathrm{M}$ bases ensuring at least 200 genotyped SNPs in each chunk. No chunks spanned across the centromeres. The para-autosomal and non-para-autosomal regions on chromosome $\mathrm{X}$ were handled separately. The resulting data were filtered on marker level by requiring IMPUTE's 'info' score $>0.3$ in both the KA06 and KA09 cohorts before merging. Merging of the imputed data was done using GTOOL (v0.7.5) ${ }^{56}$ requiring a dosage threshold above 0.9 in at least $95 \%$ of the individuals. The resulting merged data were further filtered using QCTOOL (v1.3) ${ }^{57}$ requiring a Bonferroni-corrected Hardy-Weinberg cutoff of 0.05 and a minor-allele frequency corresponding to at least one chromosome in the whole material. The final data set included 4,840,842 SNPs and INDELs.

ABO blood group assignment. We assigned blood groups according to the $\mathrm{ABO}$-system to our samples based on their genetic status of four genotyped SNPs (rs505922, rs8176746, rs8176704 and rs574347) in the region of the $A B O$ gene. These four SNPs allow for accurate assignment of both the A/B/O groups and subtyping of A into $\mathrm{A} 1$ and $\mathrm{A} 2$ and subtyping of $\mathrm{O}$ into $\mathrm{O} 01$ and $\mathrm{O} 02$ (ref. 58). Using this approach, we successfully assigned blood groups to $97.9 \%$ of our samples.

Statistic analyses. All statistical analysis was conducted in $\mathrm{R}^{59}$ and illustrations were produced using $\mathrm{R}$ and the Circos software ${ }^{60}$. Correlation between proteins and relevant variables was calculated separately for each measured protein by fitting a generalized linear model using the 'glm' function including all covariates simultaneously. The significance of the each covariate's contribution to the total variance was estimated using an analysis of variance approach as implemented by the 'anova.glm' function on the resulting generalized linear model. Covariates were considered significant for a specific protein if their Bonferroniadjusted $P$-values were below $0.05\left(P\right.$-value $\left.<3.16 \times 10^{-4}, 0.05 / 158\right)$. Each PEA measurement was individually adjusted for significant covariates (Supplementary Data 2) and rank-transformed to normality by using the 'rntransform' function available from the R-package GenABEL (v1.6.7) ${ }^{61}$. Correlations between pairs of PEA measurements were carried out, on the adjusted and rank-transformed values, using the 'cor' function applying Spearman's Rho statistics on pairwise complete observations.

The NSPHS is a population-based study and includes many relatives and special care has to be attributed to avoid relational biases. Therefore, all genetic association calculations were carried out using the GenABEL or ProbABEL ${ }^{61}$ software suites, which has been developed to enable statistical analyses of genetic data of related individuals. These packages includes functions for estimating the narrow-sense heritability $\left(h^{2}\right)$ and performing genetic association analyses ${ }^{2}$ by adjusting for pedigree structure. In brief, the heritability of each trait (protein abundance) is estimated using a polygenic model as implemented by the 'polygenic' method in the GenABEL R-package ${ }^{61}$. This heritability estimate represents the variance in the phenotype that is explained by genetic factors and is estimated by maximizing the likelihood of the trait-data under a polygenic model including fixed effects such as covariates and relatedness among individuals (kinship). The result of the 'polygenic'-call contains the inverse variance-covariance matrix of the estimates and trait residuals and is included in the downstream association calculations together with the posterior genotypic probabilities. Specifically, these calculations are performed using the ProbABEL programme using the '--mmscore' option. Kinship matrix calculations were carried out using the autosomal markers shared $(n=182,916)$ between the two types of genotyping arrays used in the KA06 and KA09 cohorts. Contribution of single SNP's to phenotypic variation on the unadjusted ddCq values was calculated in $\mathrm{R}$ by fitting a linear model (using 'Im') with ddCq values as response and the posterior genotypic probabilities as terms and fraction of variance explained was determined from the resulting model using 'summary.lm'. Fraction of variance explained by a single SNP in the adjusted phenotypes including effects of relatedness was estimated by dividing the resulting chi-square test score (from ProbABEL) with the number of samples used.

The KA06 cohort was used as discovery cohort in the GWAS and KA09 as replication cohort. As we cannot rule out protein degradation effects due to differences in storage time between the two cohorts, this split is favourable to a random split where degradation effects could affect the association analysis. Strict Bonferroni-adjusted $P$-values $\left(P\right.$-value $\left.<1.03 \times 10^{-8}, 0.05 / 4,840,842\right)$ were used to report significance in the discovery cohort and the replication cohort ( $P$-value $<0.05 /$ number of significant SNPs in the discovery cohort). We also ran a combined analysis with the same cutoff used as in the discovery phase. For all proteins with replicated hits, a conditional analysis was carried out in which the genetic associations were re-calculated using the dosage values of the top-ranking SNP as covariate. This analysis was only run in the combined material and on chromosomes that had hits in that replicated in the discovery-replication phase and $P$-value $<5 \times 10^{-8}$ was used as cutoff. 


\section{References}

1. Prensner, J. R., Rubin, M. A., Wei, J. T. \& Chinnaiyan, A. M. Beyond PSA: the next generation of prostate cancer biomarkers. Sci. Trans. Med. 4, 127rv123 (2012).

2. Scholler, N. \& Urban, N. CA125 in ovarian cancer. Biomark. Med. 1, 513-523 (2007).

3. Yue, C. et al. The effects of adalimumab and methotrexate treatment on peripheral Th17 cells and IL-17/IL-6 secretion in rheumatoid arthritis patients. Rheumatol. Int. 30, 1553-1557 (2010).

4. Hanahan, D. \& Weinberg, R. A. Hallmarks of cancer: the next generation. Cell 144, 646-674 (2011).

5. Cao, Y., DePinho, R. A., Ernst, M. \& Vousden, K. Cancer research: past, present and future. Nat. Rev. Cancer 11, 749-754 (2011).

6. Brooks, J. D. Translational genomics: The challenge of developing cancer biomarkers. Genome Res. 22, 183-187 (2012).

7. Schwanhausser, B. et al. Global quantification of mammalian gene expression control. Nature 473, 337-342 (2011).

8. Johansson, A. et al. Identification of genetic variants influencing the human plasma proteome. Proc. Natl Acad. Sci. USA 110, 4673-4678 (2013).

9. Lundberg, M., Eriksson, A., Tran, B., Assarsson, E. \& Fredriksson, S. Homogeneous antibody-based proximity extension assays provide sensitive and specific detection of low-abundant proteins in human blood. Nucleic Acids Res. 39, e102 (2011)

10. Assarsson, E. et al. Homogenous 96-Plex PEA immunoassay exhibiting high sensitivity, specificity, and excellent scalability. PLoS ONE 9, e95192 (2014).

11. Melzer, D. et al. A genome-wide association study identifies protein quantitative trait loci (pQTLs). PLoS Genet. 4, e1000072 (2008).

12. Gao, Y. C., Yuan, Z. B., Yang, Y. D. \& Lu, H. K. Effect of freeze-thaw cycles on serum measurements of AFP, CEA, CA125 and CA19-9. Scand. J. Clin. Lab. Invest. 67, 741-747 (2007).

13. Besingi, W. \& Johansson, A. Smoke related DNA methylation changes in the etiology of human disease. Hum. Mol. Genet. 23, 2290-2297 (2014).

14. Kiechl, S. et al. Association of variation at the ABO locus with circulating levels of soluble intercellular adhesion molecule-1, soluble P-selectin, and soluble E-selectin a meta-analysis. Circ. Cardiovasc. Gene 4, 681-U436 (2011).

15. Qi, L. et al. Genetic variants in ABO blood group region, plasma soluble E-selectin levels and risk of type 2 diabetes. Hum. Mol. Genet. 19, 1856-1862 (2010).

16. Feng, Y. et al. Expression of VEGF-C and VEGF-D as significant markers for assessment of lymphangiogenesis and lymph node metastasis in non-small cell lung cancer. Anat. Rec. 293, 802-812 (2010)

17. Stacker, S. A., Achen, M. G., Jussila, L., Baldwin, M. E. \& Alitalo, K. Lymphangiogenesis and cancer metastasis. Nat. Rev. Cancer. 2, 573-583 (2002)

18. Bolstad, N., Oijordsbakken, M., Nustad, K. \& Bjerner, J. Human epididymis protein 4 reference limits and natural variation in a Nordic reference population. Tumour Biol. 33, 141-148 (2012).

19. Barbosa-Morais, N. L. et al. The evolutionary landscape of alternative splicing in vertebrate species. Science 338, 1587-1593 (2012)

20. Derrien, T. et al. The GENCODE v7 catalog of human long noncoding RNAs: analysis of their gene structure, evolution, and expression. Genome Res. 22, 1775-1789 (2012)

21. Asmann, Y. W. et al. Detection of redundant fusion transcripts as biomarkers or disease-specific therapeutic targets in breast cancer. Cancer Res. 72, 1921-1928 (2012).

22. Fredrickson, B. L. et al. A functional genomic perspective on human well-being. Proc. Natl Acad. Sci. USA 110, 13684-13689 (2013).

23. Schadt, E. E. et al. Mapping the genetic architecture of gene expression in human liver. PLoS Biol. 6, e107 (2008).

24. Stranger, B. E. et al. Population genomics of human gene expression. Nat Genet. 39, 1217-1224 (2007).

25. Damas, J. K. et al. Enhanced levels of CCL19 in patients with advanced acquired immune deficiency syndrome (AIDS). Clin. Exp. Immunol. 167, 492-498 (2012)

26. Pare, G. et al. Novel association of ABO histo-blood group antigen with soluble ICAM-1: results of a genome-wide association study of 6,578 women. PLoS Genet. 4, e1000118 (2008)

27. Antonelli, A. et al. Chemokine (C-X-C motif) ligand (CXCL) 10 in autoimmune diseases. Autoimmun. Rev. 13, 272-280 (2014)

28. Antonelli, A. et al. High serum levels of CXC (CXCL10) and CC (CCL2) chemokines in untreated essential hypertension. Int. J. Immunopathol. Pharmacol. 25, 387-395 (2012).

29. WHO. Fact sheet No 297 (http://www.who.int/mediacentre/factsheets/fs297/ en/) (2013).

30. Boye, K. et al. EMMPRIN is associated with S100A4 and predicts patient outcome in colorectal cancer. Br. J. Cancer 107, 667-674 (2012).
31. Neurath, M. F. \& Finotto, S. IL-6 signaling in autoimmunity, chronic inflammation and inflammation-associated cancer. Cytokine Growth Factor Rev. 22, 83-89 (2011).

32. Rosman, Z., Shoenfeld, Y. \& Zandman-Goddard, G. Biologic therapy for autoimmune diseases: an update. BMC Med. 11, 88 (2013).

33. Pers, Y. M. et al. Predictors of response and remission in a large cohort of rheumatoid arthritis patients treated with tocilizumab in clinical practice. Rheumatology 53, 76-84 (2014).

34. Antonelli, A. et al. Interferon-gamma-inducible alpha-chemokine CXCL10 involvement in Graves' ophthalmopathy: modulation by peroxisome proliferator-activated receptor-gamma agonists. J. Clin. Endocrinol. Metab. 91 614-620 (2006)

35. Zhao, S. X. et al. Robust evidence for five new Graves' disease risk loci from staged genome-wide association analysis. Hum. Mol. Genet. 22, 3347-3362 (2013).

36. Chu, X. et al. A genome-wide association study identifies two new risk loci for Graves' disease. Nat. Genet. 43, 897-901 (2011).

37. Gallup. GALLUP POLL SOCIAL SERIES: CONSUMPTION HABITS (http:// www.gallup.com/poll/156833/one-five-adults-smoke-tied-time-low.aspx) (2012).

38. Marcus, C. S., Maxwell, G. L., Darcy, K. M., Hamilton, C. A. \& McGuire, W. P. Current approaches and challenges in managing and monitoring treatment response in ovarian cancer. J. Cancer 5, 25-30 (2014).

39. Takayama, R. et al. Serum tumor antigen REG4 as a diagnostic biomarker in pancreatic ductal adenocarcinoma. J. Gastroenterol. 45, 52-59 (2010).

40. Lango Allen, H. et al. Hundreds of variants clustered in genomic loci and biological pathways affect human height. Nature 467, 832-838 (2010).

41. Teslovich, T. M. et al. Biological, clinical and population relevance of 95 loci for blood lipids. Nature 466, 707-713 (2010).

42. le Coutre, P. et al. In vivo eradication of human BCR/ABL-positive leukemia cells with an ABL kinase inhibitor. J. Natl Cancer I 91, 163-168 (1999).

43. Chin, L., Andersen, J. N. \& Futreal, P. A. Cancer genomics: from discovery science to personalized medicine. Nat. Med. 17, 297-303 (2011).

44. Johansson, A. et al. Common variants in the JAZF1 gene associated with height identified by linkage and genome-wide association analysis. Hum. Mol. Genet. 18, 373-380 (2009).

45. Igl, W., Johansson, A. \& Gyllensten, U. The Northern Swedish Population Health Study (NSPHS)--a paradigmatic study in a rural population combining community health and basic research. Rural Remote Health 10, 1363 (2010).

46. Enroth, S., Dahlbom, I., Hansson, T., Johansson, A. \& Gyllensten, U. Prevalence and sensitization of atopic allergy and coeliac disease in the Northern Sweden Population Health Study. Int. J. Circumpolar Health 72 (2013).

47. Igl, W. et al. Animal source food intake and association with blood cholesterol, glycerophospholipids and sphingolipids in a northern Swedish population. Int J. Circumpolar. Health 72 (2013).

48. World Medical Association Declaration of Helsinki: ethical principles for medical research involving human subjects. JAMA 284, 3043-3045 (2000).

49. Mascalzoni, D. et al. Comparison of participant information and informed consent forms of five European studies in genetic isolated populations. Eur. J. Hum. Genet. 18, 296-302 (2010).

50. Purcell, S. et al. PLINK: a tool set for whole-genome association and population-based linkage analyses. Am. J. Hum. Genet. 81, 559-575 (2007).

51. Glodzik, D. et al. Inference of identity by descent in population isolates and optimal sequencing studies. Eur. J. Hum. Genet. 21, 1140-1145 (2013).

52. Genomes Project, C. et al. A map of human genome variation from populationscale sequencing. Nature 467, 1061-1073 (2010).

53. IMPUTE2 (https://mathgen.stats.ox.ac.uk/impute/impute_v2.html\#reference) (2012)

54. Delaneau, O., Zagury, J. F. \& Marchini, J. Improved whole-chromosome phasing for disease and population genetic studies. Nat. Methods 10, 5-6 (2013).

55. Howie, B., Fuchsberger, C., Stephens, M., Marchini, J. \& Abecasis, G. R. Fast and accurate genotype imputation in genome-wide association studies through pre-phasing. Nat. Genet. 44, 955-959 (2012).

56. Freeman, C. \& Marchini, J. GTOOL (http://www.well.ox.ac.uk/ cfreeman/ software/gwas/gtool.html) (2013).

57. Band, G. \& Marchini, J. QCTOOL (http://www.well.ox.ac.uk/ gav/qctool/) (2013).

58. Wolpin, B. M. et al. Variant $\mathrm{ABO}$ blood group alleles, secretor status, and risk of pancreatic cancer: results from the pancreatic cancer cohort consortium. Cancer Epidemiol Biomark. Prevent. 19, 3140-3149 (2010).

59. R Develpment Core Team. R: A Language and Environment for Statistical Computing (R Foundation for Statistical Computing, 2012).

60. Krzywinski, M. et al. Circos: an information aesthetic for comparative genomics. Genome Res. 19, 1639-1645 (2009). 
61. Aulchenko, Y. S., Ripke, S., Isaacs, A. \& Van Duijn, C. M. GenABEL: an R library for genome-wide association analysis. Bioinformatics. 23, 1294-1296 (2007).

62. Chen, W. M. \& Abecasis, G. R. Family-based association tests for genomewide association scans. Am. J. Hum. Genet. 81, 913-926 (2007).

\section{Acknowledgements}

We are grateful for the contribution of district nurse Svea Hennix for data collection and Inger Jonasson for logistics and coordination of the health survey. We also thank all the participants from the community for their interest and willingness to contribute to this study. Illumina genotyping was performed by the SNP\&SEQ Technology Platform in Uppsala, Sweden. Whole-Exome Sequencing was performed by the Uppsala Genome Center Facility in Uppsala, Sweden. PEA measurements were carried out by the PLA-based profiling facility in Uppsala, Sweden. The computations were performed on resources provided by SNIC through Uppsala Multidisciplinary Center for Advanced Computational Science (UPPMAX) under projects b2011203 and p2013140. The Northern Swedish Population Health Study (NSPHS) was funded by the Swedish Medical Research Council (Project Number K2007-66X-20270-01-3, 2011-5252, 2012-2884 and 2011-2354), the Foundation for Strategic Research (SSF). NSPHS as part of EUROSPAN (European Special Populations Research Network) was also supported by the European Commission FP6 STRP grant number 01947 (LSHG-CT-2006-01947). This work has also been supported by the Swedish Society for Medical Research (SSMF). The PEA measurements were performed by the Science for Life Laboratory, Clinical Biomarker Facility. We are grateful for the support of Associate Professor Masood Kamali-Moghaddam and Professor Agneta Siegbahn at this facility.

\section{Author contributions}

S.E. designed experiments, developed analysis tools, analysed data and wrote the paper. Å.J. contributed to the design of the experiments. S.B.E. interpreted results. U.G. conceived of the study, designed the experiments and wrote the paper.

\section{Additional information}

Supplementary Information accompanies this paper at http://www.nature.com/ naturecommunications

Competing financial interests: Ulf Gyllensten and Stefan Enroth are authors on a patent application entitled 'Determination and analysis of Biomarkers in clinical samples'; serial number GB1410956.5 (2014). The remaining authors declare no competing financial interests.

Reprints and permission information is available online at http://npg.nature.com/ reprintsandpermissions/

How to cite this article: Enroth, S. et al. Strong effects of genetic and lifestyle factors on biomarker variation and use of personalized cutoffs. Nat. Commun. 5:4684 doi: $10.1038 /$ ncomms5684 (2014).

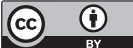

This work is licensed under a Creative Commons Attribution 4.0 International License. The images or other third party material in this article are included in the article's Creative Commons license, unless indicated otherwise in the credit line; if the material is not included under the Creative Commons license, users will need to obtain permission from the license holder to reproduce the material. To view a copy of this license, visit http://creativecommons.org/licenses/by/4.0/ 